\title{
Meal Data Name
}

National Cancer Institute

\section{Source}

National Cancer Institute. Meal Data Name. NCI Thesaurus. Code C162140.

Verbatim food product name that is either pre-printed or collected on a CRF. 